\title{
Disruption of the psychiatric risk gene Ankyrin 3 enhances microtubule dynamics through GSK3/CRMP2 signaling
}

Jacob C. Garza ${ }^{1,2,3}$, Xiaoli Qi ${ }^{1,2,3}$, Klaudio Gjeluci ${ }^{1,3,4}$, Melanie P. Leussis ${ }^{1,5}$, Himanish Basu ${ }^{6,7}$, Surya A. Reis ${ }^{1}$, Wen Ning Zhao ${ }^{1}$, Nicolas H. Piguel ${ }^{8}$, Peter Penzes ${ }^{8}$, Stephen J. Haggarty ${ }^{1}$, Gerard J. Martens ${ }^{9}$, Geert Poelmans ${ }^{9,10}$ and Tracey L. Petryshen 1,2,3

\begin{abstract}
The ankyrin 3 gene (ANK3) is a well-established risk gene for psychiatric illness, but the mechanisms underlying its pathophysiology remain elusive. We examined the molecular effects of disrupting brain-specific Ank3 isoforms in mouse and neuronal model systems. RNA sequencing of hippocampus from Ank3+/- and Ank3+/+ mice identified altered expression of 282 genes that were enriched for microtubule-related functions. Results were supported by increased expression of microtubule end-binding protein 3 (EB3), an indicator of microtubule dynamics, in Ank3+/mouse hippocampus. Live-cell imaging of EB3 movement in primary neurons from Ank3+/- mice revealed impaired elongation of microtubules. Using a CRISPR-dCas9-KRAB transcriptional repressor in mouse neuro-2a cells, we determined that repression of brain-specific Ank3 increased EB3 expression, decreased tubulin acetylation, and increased the soluble:polymerized tubulin ratio, indicating enhanced microtubule dynamics. These changes were rescued by inhibition of glycogen synthase kinase 3 (GSK3) with lithium or CHIR99021, a highly selective GSK3 inhibitor. Brain-specific Ank3 repression in neuro-2a cells increased GSK3 activity (reduced inhibitory phosphorylation) and elevated collapsin response mediator protein 2 (CRMP2) phosphorylation, a known GSK3 substrate and microtubule-binding protein. Pharmacological inhibition of CRMP2 activity attenuated the rescue of EB3 expression and tubulin polymerization in Ank3-repressed cells by lithium or CHIR99021, suggesting microtubule instability induced by Ank3 repression is dependent on CRMP2 activity. Taken together, our data indicate that ANK3 functions in neuronal microtubule dynamics through GSK3 and its downstream substrate CRMP2. These findings reveal cellular and molecular mechanisms underlying brain-specific ANK3 disruption that may be related to its role in psychiatric illness.
\end{abstract}

\section{Introduction}

Large-scale genomic studies are providing a clearer picture of the genetic architecture of psychiatric illness. Genetic variation in ANK3 is associated with several psychiatric disorders, including bipolar disorder (BD) and autism spectrum disorders $(\mathrm{ASD})^{1-11}$. Human postmortem brain studies demonstrate that carriers of ANK3

\footnotetext{
Correspondence: Tracey L. Petryshen (tpetryshen@mgh.harvard.edu) ${ }^{1}$ Center for Genomic Medicine, Massachusetts General Hospital, Boston, MA, USA

${ }^{2}$ Department of Psychiatry, Harvard Medical School, Boston, MA, USA Full list of author information is available at the end of the article.
}

alleles associated with $\mathrm{BD}$ have lower $A N K 3$ expression at the transcript and protein levels ${ }^{12,13}$, suggesting that decreased expression of ANK3 contributes to disease. Despite strong genetic evidence that $A N K 3$ contributes to psychiatric illness ${ }^{14}$, the precise mechanism is unknown.

ANK3 encodes the ankyrin-G scaffolding protein that anchors integral membrane proteins to the cytoskele$\operatorname{ton}^{15,16}$. There are several protein isoforms of ankyrin-G due to alternative splicing and alternative starting exons $^{13,17}$. These isoforms have unique functions and tissue distribution, including isoforms that are only expressed in brain. Genomic regions associated with BD 
span exon 1b of $A N K 3$ and the intron upstream of exon 37 , exons that are only present in brain-specific isoforms. Furthermore, rare mutations identified in ASD patients are predominantly located within the brain-specific exon $37^{1-8}$. The brain-specific isoforms are primarily known for their function in formation of the neuron axon initial segment (AIS) and clustering of ion channels at the nodes of Ranvier along axons ${ }^{18,19}$. Interestingly, BD patients carrying a BD-associated risk allele for ANK3 have a decreased fractional anisotropy in the uncinate fasciculus, which is an indication of impaired axon function or axonal damage in these forebrain connections ${ }^{11}$.

Recent evidence implicates cytoskeleton dysfunction in psychiatric illness ${ }^{20-22}$. Microtubules are components of the cytoskeleton that contribute to the morphology of axons and dendrites in neurons, and facilitate transport of cellular cargo. They are composed of $\alpha$ and $\beta$ tubulin heterodimers that continuously polymerize and depolymerize at the microtubule plus end (i.e., microtubule dynamics), leading to continuous growth and shrinkage of microtubules ${ }^{23}$. ANK3 is reported to bind microtubules directly or through binding of microtubule-associated proteins at the plus end stabilizing $\mathrm{cap}^{24-26}$ that prevents depolymerization. The interaction between ANK3 and microtubules provides a biological basis for examining brain-specific $A n k 3$ in the regulation of microtubule dynamics.

We have previously demonstrated that brain-specific Ank3 isoforms regulate psychiatric-related behaviors in mice, and alterations in these behaviors in $A n k 3+1-$ mice are reversed by the mood stabilizer lithium ${ }^{27,28}$. An important target of lithium is GSK3, which is implicated in psychiatric illness by animal studies ${ }^{29,30}$. Among the downstream substrates of GSK3, CRMP2 has emerged as a prime target for regulation of microtubule dynamics and stability $^{31-33}$. In its unphosphorylated state, CRMP2 binds tubulin heterodimers and stabilizes the plus end of microtubules ${ }^{34}$; however, upon phosphorylation by GSK3, CRMP2 activity is suppressed and binding to microtubules is reduced. In Caenorhabditis elegans, the homologs of ANK3 (UNC-44) and CRMP2 (UNC-33) are required to organize microtubules in neurons ${ }^{35}$. Therefore, it is possible that ANK3 modulates microtubule dynamics through a mechanism involving CRMP2 signaling.

In the current study, we investigated the molecular impact of reducing expression of brain-specific Ank3 isoforms, based on the patient genetic and expression studies noted above that implicate reduced expression of these isoforms in disease. Using RNA sequencing, biochemical, and live cell-imaging methods in mouse and neuronal model systems, we determined that brainspecific Ank3 deficiency is associated with enhanced microtubule dynamics (i.e., increased tubulin polymerization/depolymerization). Furthermore, we demonstrate that microtubule changes induced by brainspecific Ank3 repression are rescued by lithium or a selective inhibitor of GSK3 through a CRMP2-dependent mechanism. Our findings establish for the first time that brain-specific Ank3 is important for maintaining proper microtubule dynamics through GSK3/CRMP2 signaling.

\section{Materials and Methods}

See Supplementary Information for detailed methods.

\section{Animals}

Male Ank3+/- mice with heterozygous disruption of Ank3 exon $1 \mathrm{~b}^{18}$ were crossed to female C57BL/6J mice (Jackson Laboratory, Bar Harbor, ME) to generate Ank3 $+/-$ and $A n k 3+I+$ progeny. Experiments were conducted in accordance with the National Institutes of Health guidelines and approval of the Institutional Animal Care and Use Committees of Massachusetts Institute of Technology and Massachusetts General Hospital.

\section{RNA sequencing and data analysis}

Hippocampal RNA from 10 male 16-20wk old mice per genotype was pooled for RNA sequencing. Trimmed sequence reads were aligned onto the Mus musculus GRCm38/mm10 genome and analyzed using the Tuxedo package within the GenePattern platform (https:// genepattern.broadinstitute.org) ${ }^{36}$. Differentially expressed genes were identified based on minimum 1.2-fold change and uncorrected $P \leq 1 \times 10^{-3}$. The Ingenuity Pathway Analysis package was used to identify overrepresented biological pathways, with a focus on 'Canonical Pathways' and 'Diseases and Functions'.

\section{Live cell imaging}

Mouse primary forebrain neurons were generated from P0 Ank3+/+ and Ank3+/- mice. Cells were transfected with mPA-GFP-EB3-7 (Addgene, Cambridge, MA) at DIV11-12 and imaged at DIV14. Axon segments $70-150 \mu \mathrm{m}$ in length were imaged starting $\sim 60 \mu \mathrm{m}$ from the soma at $2 \mathrm{~s}$ intervals for $300 \mathrm{~s}$. EB3 comet trajectory was manually traced from kymographs generated using the ImageJ Kymolyzer macro ${ }^{37}$ to calculate comet length, duration, and velocity.

\section{Western blot}

Protein lysates from neuro-2a cells or hippocampal tissue from male mice were separated by SDS-PAGE and blotted onto PVDF membranes, probed with specific primary antibodies and HRP-linked secondary antibodies (Supplementary Table 1), followed by electrochemiluminescent detection. Protein expression was quantified by normalizing to GAPDH, and 
phosphorylated or acetylated protein expression by normalizing to the corresponding total protein.

\section{CRISPR-mediated Ank3 transcriptional repression}

Single guide RNA (sgRNA) sequences were designed to target mouse Ank3 exon 1b using the CRISPR Design Tool (http://crispr.mit.edu) (Supplementary Table 2). Ank3-targeting and non-targeting control sgRNAs were cloned into the sgRNA(MS2)-EF1 $\alpha$ plasmid (gift from Dr. Feng Zhang) ${ }^{38}$.

\section{Cell culture}

Mouse neuro-2a cells (ATCC, Manassas, VA) were dual transfected with the pHAGE-EF1 $\alpha$-dCas9-KRAB transcriptional repressor plasmid ${ }^{39}$ (Addgene) and sgRNA (MS2)-EF1 $\alpha$ plasmid expressing Ank3-targeting or control sgRNA. For drug experiments, cells were treated for $1 \mathrm{~h}$ with the GSK3 inhibitors lithium (Sigma-Aldrich, St. Louis, Mo) or CHIR99021 (LC Laboratories, Woburn, $\mathrm{Ma})$, or for $24 \mathrm{~h}$ with the CRMP2 inhibitor lacosamide (Sigma-Aldrich, St. Louis, Mo).

\section{RT-qPCR}

SYBR Green qPCR was performed using $1 \mu \mathrm{g}$ cDNA using gene and Ank3 isoform-specific primers (Supplementary Table 3$)^{40}$. Expression was normalized to beta-2microglobulin.

\section{Tubulin polymerization assay}

Neuro-2a cells were lysed to obtain soluble and insoluble protein fractions for western blot detection of $\alpha$ tubulin $^{41,42}$.

\section{Statistical analysis}

Statistical analyses were performed by unpaired Student's $t$ test, or one- or two-way ANOVA followed by post hoc tests, using StatView version 5 or SPSS version 16 . Sample sizes were determined based on previously published literature and our preliminary data. Significance threshold was set at $P<0.05$.

\section{Results}

RNA sequencing identifies expression changes in microtubule-related pathways in Ank3+I- mouse hippocampus

RNA sequencing analysis was performed to identify genes with altered expression in hippocampus from Ank3+/mice, which exhibit $50 \%$ reduced expression of brainspecific Ank3 compared to wild-type Ank3+/+ mice (Supplementary Figure 1). On the basis of our targeted read depth of 25 million reads, we expected the RNA sequencing analysis to detect predominantly abundant genes. A total of 282 genes were significantly differentially expressed (fold change $\geq 1.2$, uncorrected $P \leq 1 \times 10^{-3}$ ) between $A n k 3+/-$ and $A n k 3+/+$ hippocampus (102 upregulated, 180 downregulated; Supplementary Table 4). Ingenuity Pathway Analysis of the differentially expressed genes identified significant overrepresentation of the Axonal Guidance Signaling canonical pathway (17 genes; corrected $P=$ 0.0088). Among the Disease \& Function pathways, the Microtubule Dynamics pathway was most significantly enriched (34 genes; uncorrected $P=6.7 \times 10^{-5}$ ). Ten of the 17 Axonal Guidance Signaling genes were also annotated as Microtubule Dynamics genes. The Microtubule Dynamics pathway is categorized within a higher-level function of Cellular Assembly and Organization, which contains three other pathways that were overrepresented among the 282 differentially expressed genes, although with weaker statistical evidence: Fusion of Vesicles (6 genes, $P=2.0 \times 10^{-4}$ ), Organization of Cytoskeleton (36 genes, $P=3.1 \times 10^{-4}$ ), and Extension of Cellular Protrusions (10 genes, $P=3.3 \times$ $\left.10^{-4}\right)$. Many of the genes in the latter three pathways overlap the Microtubule Dynamics and Axonal Guidance Signaling pathways, resulting in a total of 47 genes across the five pathways (Table 1). As the identified pathways represent cellular functions requiring microtubules, we focused subsequent experiments on the role of $A n k 3$ in microtubule dynamics.

\section{Enhanced microtubule dynamics in Ank3+I- mouse hippocampus}

To obtain support for microtubule defects in Ank3+/mouse brain, we evaluated the expression of the EB3 endbinding protein. EB3 modulates stability at the microtubule plus end, serving as a marker of growing microtubules $^{43}$, and is reported to directly interact with Ank $3^{24-26}$. Western blot analysis of EB3 in hippocampus isolated from $A n k 3+/+$ and $A n k 3+/-$ mice determined that expression was increased 1.5-fold in $A n k 3+/-$ mice compared to $A n k 3+/+$ mice $(P<0.001$, Fig. 1$)$. The substantial elevation in EB3 expression suggests that reduction of brain-specific $A n k 3$ is associated with enhanced microtubule dynamics (i.e., increased polymerization/depolymerization of tubulin at the plus end).

\section{Brain-specific Ank3 reduction impairs microtubule elongation in axons}

To directly monitor the effect of Ank3 reduction on the dynamic properties of microtubules, we performed live-cell imaging of GFP-tagged EB3 in mouse primary neurons. As microtubules polymerize, EB3-GFP puncta at the plus end cap appear as mobile comets, which dissipate when the microtubules depolymerize ${ }^{44}$. We analyzed EB3 comet length, duration, and velocity in axons of DIV14 primary neurons from $A n k 3+/+$ and $A n k 3+/-$ neonatal mice (Fig. 2a). The trajectory length of EB3 comets was $~ 15 \%$ shorter in axons of Ank3+I- neurons compared to Ank3+/+ neurons (Fig. 2b, $P=0.002$ ), indicating decreased 
Table 1 Microtubule-related pathways enriched for genes that are differentially expressed between Ank3+I- and Ank3+I+ mouse hippocampus

\begin{tabular}{|c|c|c|c|c|c|c|}
\hline Gene & $\begin{array}{l}\text { Fold change } \\
(\text { Ank } 3+I-\text { vs } \\
\text { Ank } 3+I+)\end{array}$ & $\begin{array}{l}\text { Axonal } \\
\text { guidance } \\
\text { signaling }\end{array}$ & $\begin{array}{l}\text { Microtubule } \\
\text { dynamics }\end{array}$ & $\begin{array}{l}\text { Fusion of } \\
\text { vesicles }\end{array}$ & $\begin{array}{l}\text { Organization } \\
\text { of } \\
\text { cytoskeleton }\end{array}$ & $\begin{array}{l}\text { Extension of } \\
\text { cellular } \\
\text { protrusions }\end{array}$ \\
\hline$A G T$ & 1.30 & & $x$ & & $x$ & $x$ \\
\hline ANKRD27 & -1.30 & & $x$ & & $x$ & $x$ \\
\hline ARHGAP33 & -1.26 & & $x$ & & $x$ & \\
\hline BAG3 & -1.48 & $x$ & & & $x$ & \\
\hline BCAR1 & -1.26 & & $x$ & & $x$ & $x$ \\
\hline$B C R$ & -1.25 & & $x$ & & $x$ & \\
\hline$B S N$ & -1.20 & & $x$ & $x$ & $x$ & \\
\hline CACNATA & -1.21 & $x$ & $x$ & & $x$ & \\
\hline CAV2 & 1.30 & & & $x$ & & \\
\hline CDK18 & -1.31 & & $x$ & & $x$ & \\
\hline DPYSL5 & -1.25 & $x$ & $x$ & & $x$ & $x$ \\
\hline$D S P$ & 1.26 & & $x$ & & $x$ & \\
\hline DVL2 & -1.40 & & $x$ & & $x$ & \\
\hline E2F4 & -1.29 & & $x$ & & x & \\
\hline EPHB6 & -1.20 & $x$ & $x$ & & $x$ & \\
\hline FLNB & -1.24 & & & & $x$ & \\
\hline FOXO6 & -1.39 & & $x$ & & $x$ & \\
\hline GAS2L 1 & -1.25 & & $x$ & & $x$ & \\
\hline GPR116 & 1.23 & & $x$ & & $x$ & \\
\hline HDAC6 & -1.26 & & $x$ & $x$ & $x$ & \\
\hline HTR1A & -1.32 & & $x$ & & $x$ & \\
\hline$I D E$ & -1.37 & & $x$ & & $x$ & \\
\hline LAMP2 & 1.21 & & & $x$ & & \\
\hline LIMK1 & -1.24 & $x$ & $x$ & & $x$ & $x$ \\
\hline LMTK3 & -1.24 & & $x$ & & $x$ & \\
\hline$M A O A$ & 1.30 & & $x$ & & $x$ & \\
\hline MAP2K2 & -1.22 & $x$ & & & & \\
\hline MATK & -1.29 & & $x$ & & $x$ & \\
\hline MYH9 & -1.27 & & $x$ & & $x$ & $x$ \\
\hline NTNG1 & 1.40 & $x$ & $x$ & & $x$ & $x$ \\
\hline PLCB4 & 1.81 & $x$ & & & & \\
\hline PLC1 & -1.23 & $x$ & & & & \\
\hline PLCH2 & -1.22 & $x$ & & & & \\
\hline PLXNA1 & -1.21 & $x$ & & & & \\
\hline PLXND1 & -1.32 & $x$ & $x$ & & $x$ & \\
\hline PRKCD & 2.86 & $x$ & $x$ & & $x$ & \\
\hline RIMS4 & -1.48 & & $x$ & & $x$ & \\
\hline
\end{tabular}


Table 1 continued

\begin{tabular}{|c|c|c|c|c|c|c|}
\hline RRAS2 & 1.48 & $x$ & & & & \\
\hline SEMA5A & 1.24 & X & & & & \\
\hline SLIT3 & -1.21 & $x$ & $x$ & & $x$ & \\
\hline SMURF1 & -1.27 & & $x$ & & $x$ & X \\
\hline STX1A & -1.29 & & & $x$ & & \\
\hline STX8 & 1.49 & & & $x$ & & \\
\hline TGFB3 & -1.48 & & $x$ & & $x$ & \\
\hline ULK1 & -1.29 & & X & & $x$ & X \\
\hline UNC5B & -1.24 & X & X & & $x$ & $x$ \\
\hline WNTTA & -1.37 & $x$ & X & & $x$ & \\
\hline
\end{tabular}
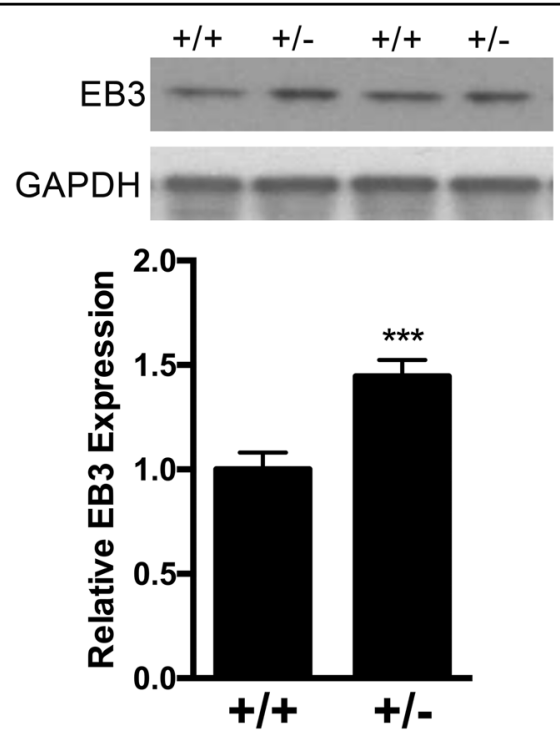

Fig. 1 Increased expression of EB3 in Ank3+/- mouse hippocampus. Top: Representative western blot of EB3 and GAPDH reference protein in hippocampal tissue from Ank3+/+ and Ank3+/mice. Bottom: Quantification of EB3 expression normalized to GAPDH expression. The data were analyzed using two-tailed Student's $t$ test, Ank3+/ $+n=12 ; A n k 3+/-n=13$. The data are presented as mean \pm s.e.m. ${ }^{* *} P<0.001$

microtubule elongation. EB3 comets in Ank3+/- axons were detected in $\sim 15 \%$ fewer frames of the $300 \mathrm{~s}$ time-lapse imaging video compared to the comets in $A n k 3+/+$ axons (Fig. 2c, $P=0.003$ ), suggesting decreased duration of EB3 bound to microtubule plus ends. The velocity of EB3 comets did not differ between $A n k 3+/-$ axons and $A n k 3+/+$ axons, indicating that the rate of microtubule polymerization was unchanged (Fig. $2 \mathrm{~d}, P>0.05$ ). These data suggest an increase in the dynamics of microtubules with an overall reduction of microtubule elongation in $A n k 3+/-$ axons compared to $A n k 3+1+$ axons.

\section{Establishment of a neuronal model of brain-specific Ank3 repression}

We established a cellular model to investigate the mechanism underlying impaired microtubule elongation associated with brain-specific $A n k 3$ reduction. Mouse neuro-2a cells were dual-transfected with a CRISPR/ dCas9 KRAB repressor and either a sgRNA targeting $A n k 3$ exon $1 \mathrm{~b}$ or a control sgRNA (Fig. 3a). Fourteen sgRNA sequences were screened for efficacy of $A n k 3$ exon1b repression (Supplementary Figure 2). One sgRNA (\#7) was selected and selective repression of Ank3 exon $1 \mathrm{~b}$ was verified (Fig. 3b; Supplementary Information). Western blot analysis indicated that $A n k 3$ repression elevated EB3 expression by $40 \%$ compared to control cells (Fig. 3c, $P<0.01$ ). This aligns with our earlier observation of increased EB3 expression in hippocampus from $A n k 3$ $+/-$ mice compared to $A n k 3+/+$ mice (Fig. 1), thereby validating our neuronal model system.

\section{Repression of brain-specific Ank3 in cells reduces polymerized tubulin}

To assess the enhancement of microtubule dynamics associated with brain-specific Ank3 repression, we examined characteristics of tubulin (i.e., acetylation and polymerization state) in our neuronal model system. Tubulin acetylation is an indicator of the overall stability of microtubules, such that microtubules that are more stable and resistant to turnover have higher acetylation levels, whereas more microtubules that are more dynamic and susceptible to turnover have lower acetylation levels ${ }^{45,46}$. Western blot analysis revealed a $45 \%$ reduction 

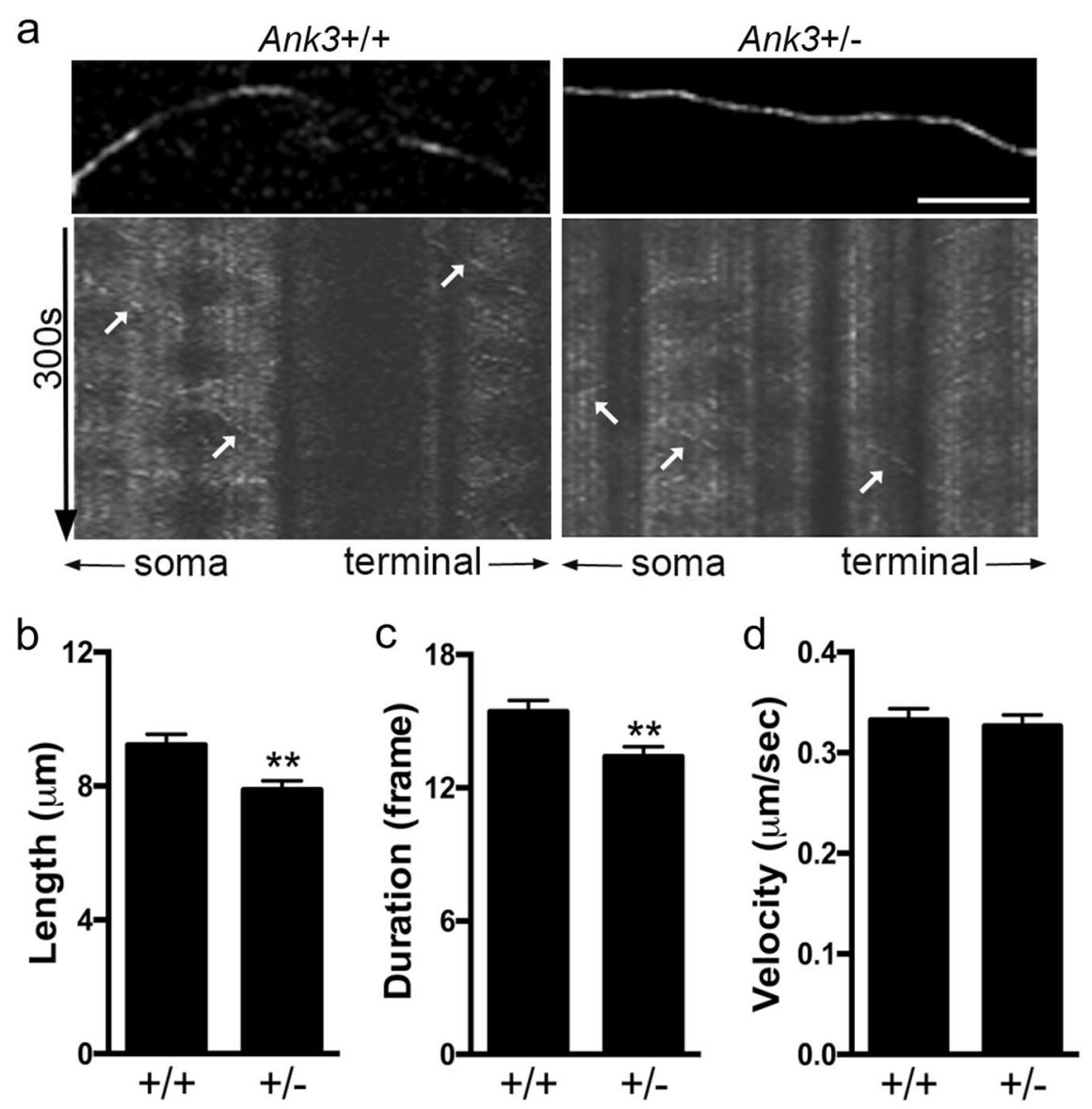

Fig. 2 Reduced brain-specific Ank3 expression is associated with enhanced microtubule dynamics in axons of mouse primary neurons. a Representative kymographs from live-cell imaging of EB3 comets in DIV14 primary neurons from Ank3+/+ (left) and Ank3+/- (right) P0 neonatal mice. Top: EB3-GFP puncta present in the axon. Scale bar $=10 \mu \mathrm{m}$. Bottom: Kymographs show the movement of EB3 comets. The $x$-axis represents the position along the axon and the $y$-axis represents time $(300 \mathrm{~s})$. White arrows indicate representative comet traces. Quantification of EB3 comet (b) length, (c) duration, and (d) velocity. The data were collected from three independent experiments. Ank $3+/+, n=245$ comets from 27 axons of 6 mice; Ank3+/-, $n=220$ comets from 14 axons of 7 mice. The data were analyzed using two-tailed Student's $t$ test. The data are presented as mean \pm s.e.m. ${ }^{* *} P<0.01$

of acetylated tubulin normalized to total tubulin in Ank3repressed cells compared to control cells (Fig. $3 \mathrm{~d}, P<$ 0.05), suggesting microtubules are more dynamic when Ank3 is repressed. Further, western blot analysis of tubulin in soluble and polymerized protein fractions ${ }^{41,47-}$ 49 determined that brain-specific Ank3 repression led to an increase in the amount of tubulin in the soluble protein fraction and a concomitant decrease in the polymerized fraction compared to control cells. This resulted in a 1.4fold increase in the ratio of soluble to polymerized tubulin (Fig. 3e, $P<0.01$ ), indicating a shift in equilibrium from microtubule-associated tubulin towards free tubulin as a result of brain-specific $A n k 3$ repression. This shift was not due to changes in total tubulin expression between Ank3repressed and control cells (Fig. 3e, $P>0.05$ ). These results are consistent with increased turnover at the microtubule plus ends due to repression of brain-specific Ank3.

\section{GSK3 inhibition rescues microtubule changes associated} with Ank3 repression

To examine the molecular mechanisms underlying enhanced microtubule dynamics in our neuronal model system, we investigated the effects of lithium treatment, which we previously demonstrated reverses behavioral abnormalities of brain-specific $A n k 3+/-$ mice $^{27,28}$. In addition, to determine whether any effects of lithium are mediated by its known target GSK3, we also investigated CHIR99021, a highly selective inhibitor of both GSK3 $\alpha$ and GSK $3 \beta^{50}$. Control and $A n k 3$ exon 1b repressed neuro-2a cells treated with vehicle, $1 \mathrm{mM}$ lithium chloride, or $1 \mu \mathrm{M}$ CHIR99021 for $1 \mathrm{~h}$ (Fig. 4a) were assessed for EB3 expression and tubulin polymerization. While Ank3 repression increased EB3 expression by 50\% compared to control in vehicle-treated cells (Fig. 4b, post hoc $P<$ 0.01 ), as expected based on our previous experiment (Fig. 3e), the increase was attenuated by lithium and 

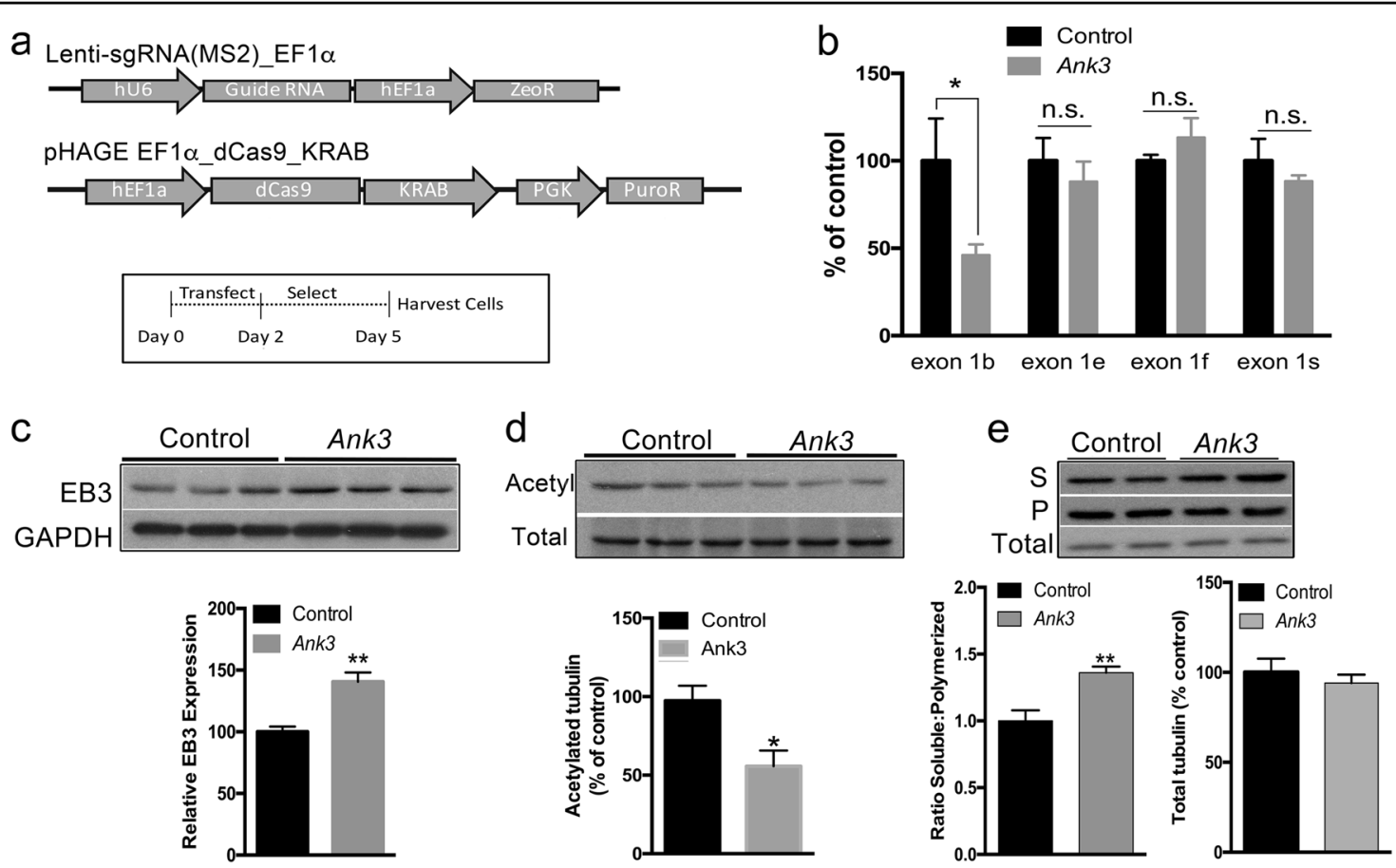

Fig. 3 CRISPR/dCas9 mediated repression of brain-specific Ank3 in mouse neuro-2a cells. a Top: Schematic representation of the plasmids used for repression of brain-specific Ank3. pHAGE-EF1a_dCas9_KRAB expressed the deactivated Cas9 fused to the KRAB transcriptional repressor. Lenti-sgRNA(MS2)_EF1a expressed the control sgRNA or an sgRNA targeting Ank3 exon 1b. See Supplementary Figure 2 for qPCR screening of sgRNA sequences for efficiency of Ank3 exon 1b repression. Bottom: Schematic representation of the experimental design. Neuro-2a cells were transfected with the two plasmids, followed by puromycin and zeocin selection, and cell harvest for protein extraction. $\mathbf{b}$ Expression of Ank3 exon 1b, exon 1e, exon $1 \mathrm{f}$, and exon $1 \mathrm{~s}$ was measured by qPCR using isoform-specific primers in neuro-2a cells expressing the control sgRNA or the Ank3-targeting sgRNA. Expression was normalized to beta-2-microglobulin and presented as percent of the control sgRNA for each starting exon. c Transcriptional repression of brain-specific Ank3 in neuro-2a cells alters EB3 expression. Top: Representative western blots of EB3 and GAPDH. Bottom: Quantification of EB3 expression normalized to GAPDH. $\mathbf{d}$ Transcriptional repression of brain-specific Ank3 in neuro-2a cells alters acetylation of a-tubulin. Top: Representative western blots of acetylated a-tubulin (Acetyl) and total a-tubulin (Total). Bottom: Quantification of acetylated a-tubulin expression normalized to total tubulin. e Transcriptional repression of brain-specific Ank3 in neuro-2a cells alters the ratio of soluble:polymerized tubulin. Top: Representative western blots of a-tubulin in soluble (S) and polymerized (P) protein fractions, and in total cell lysate (Total). Bottom left: Quantification of the ratio of soluble:polymerized tubulin. Bottom right: Quantification of total tubulin normalized to GAPDH. Western blot data were averaged from three independent experiments with three biological replicates per group in each experiment. Control, non-targeting sgRNA; Ank3, sgRNA targeting Ank3 exon 1b. Data were analyzed using two-tailed Student's $t$ test. Data are presented as mean \pm s.e.m. ${ }^{*} P<0.05,{ }^{* *} P<0.01$, n.s. indicates not significant

CHIR99021 treatment (Fig. 4b, both post hoc $P>0.05$ ). Similarly, while Ank3 exon 1b repression increased the ratio of soluble:polymerized tubulin by 1.35 -fold compared to control in vehicle-treated cells (Fig. 4c, post hoc $P<0.01)$, the ratio was normalized by treatment with lithium or CHIR99021 (Fig. 4c, both post hoc $P>0.05$ ). To rule out the possibility that the rescue was due to normalizing expression of $A n k 3$, we confirmed that $A n k 3$ expression did not differ after treatment with lithium or CHIR99021 compared to vehicle (Supplementary Figure 3). These results indicate that GSK3 is involved in changes to microtubule dynamics induced by repression of brainspecific Ank3.

To investigate the relationship between brain-specific Ank3 and GSK3 activity, we measured phosphorylation at key regulatory sites of GSK3 $\beta$ and GSK $3 \alpha$, serine 9 (GSK3 $\beta-p S 9)$ and serine 21 (GSK3 $\alpha-\mathrm{pS} 21)$, which suppress activity ${ }^{51,52}$, and tyrosine 216 (GSK3ß-pY216) and tyrosine 279 (GSK3 $\alpha$-pY279), which promote activity in the absence of serine $9 / 21$ phosphorylation ${ }^{53}$. While repression of brain-specific Ank3 did not affect GSK3 $\beta$ pY216 or GSK3 $\alpha$-pY279 levels (Fig. 4d, both $P>0.05$ ), there were significant reductions in GSK3 $\beta$-pS9 and GSK3 $\alpha$-pS21 (Fig. 4d, both $P<0.01$ ). These data suggest that repression of brain-specific Ank3 upregulates GSK3 activity through reduced phosphorylation of GSK3 $\beta$-pS9 and GSK3 $\alpha$-pS21 regulatory sites.

\section{Brain-specific Ank3 repression enhances GSK3-mediated inhibition of CRMP2 microtubule stabilization}

The GSK3 substrate CRMP2 has a key regulatory role in microtubule dynamics by promoting microtubule stability through interactions with tubulin heterodimers and acting as an adapter with motor proteins ${ }^{33,54}$. This 


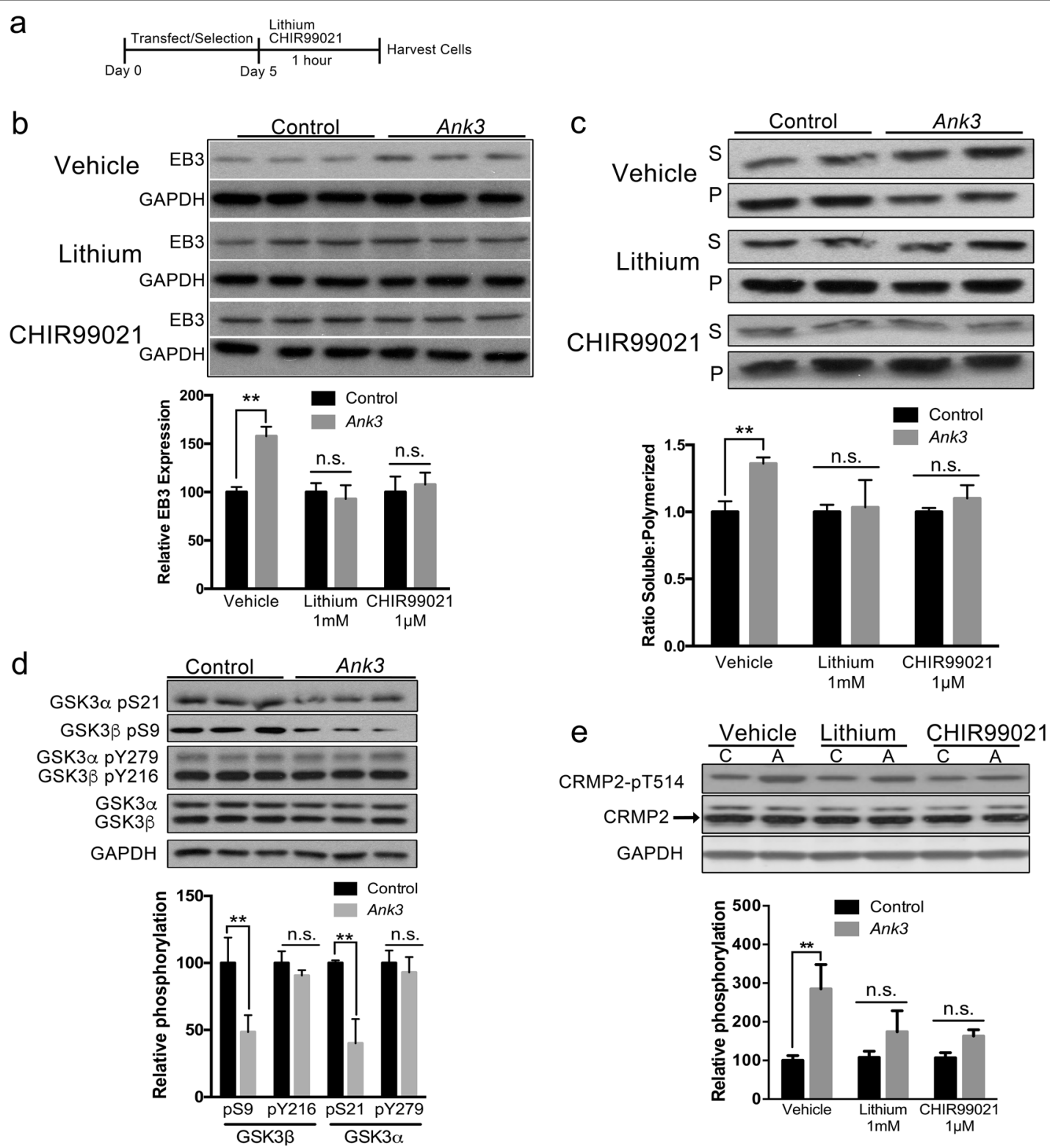

Fig. 4 Changes in EB3 expression and tubulin polymerization induced by brain-specific Ank3 repression are normalized by inhibition of GSK3. a Schematic representation of the experimental design. Mouse neuro-2a cells were transfected with pHAGE-EF1a-dCas9-KRAB repressor plasmid and sgRNA(MS2)_EF1a plasmid expressing either the non-targeting control sgRNA or the sgRNA targeting Ank3 exon 1b, followed by puromycin and zeocin selection, and treatment with lithium $(1 \mathrm{mM})$, CHIR99021 $(1 \mu \mathrm{M})$, or DMSO vehicle for $1 \mathrm{~h}$ prior to cell harvest for protein extraction. b Top: Representative western blot of EB3 and GAPDH. Bottom: Quantification of EB3 expression normalized to GAPDH. Two-way ANOVA, drug effect $F_{(2,30)}=4.217 P=0.02$, Ank3 repression effect $F_{(1,30)}=4.137 P=0.05$, interaction $F_{(2,30)}=4.217, P=0.02$. c Top: Representative western blot of a-tubulin in soluble $(S)$ and polymerized $(P)$ protein fractions. Bottom: Quantification expressed as the ratio of a-tubulin in soluble and polymerized fractions. Two-way ANOVA, drug effect $F_{(2,28)}=2.460 P=0.10$, Ank3 repression effect $F_{(1,28)}=3.281 P=0.08$, interaction $F_{(2,28)}=5.463 P$

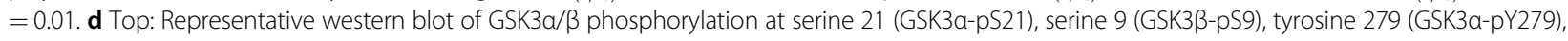

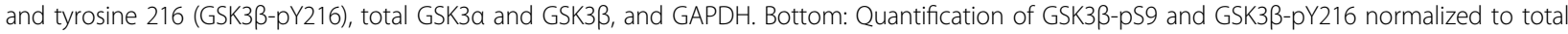
GSK3 $\beta$, and GSK3a-pS21 and GSK3a-pY279 normalized to total GSK3a. e Top: Representative western blot of CRMP2 phosphorylation at threonine 514 (CRMP2-pT514), total CRMP2, and GAPDH. Bottom: Quantification of CRMP2-pT514 normalized to total CRMP2. Two-way ANOVA, drug effect $F_{(2,12)}=4.137 P=0.05$, Ank3 repression effect $F_{(1,12)}=3.281 P=0.08$, interaction $F_{(2,12)}=4.217 P=0.02$. Western blot data were averaged from three independent experiments with three biological replicates per group in each experiment. Control or $C$, non-targeting sgRNA; Ank3 or A, sgRNA targeting Ank3 exon 1b. The data were analyzed using two-tailed Student's $t$ test or ANOVA and Bonferroni post hoc tests. Data are presented as mean \pm s.e.m. ${ }^{*} P<0.05,{ }^{*} P<0.01$. n.s. indicates not significant 
interaction is highly regulated by GSK3-dependent phosphorylation, where phosphorylation of CRMP2 threonine 514 (CRMP2-pT514) inhibits the interaction of CRMP2 with tubulin heterodimers ${ }^{33,55}$. We investigated the effect of brain-specific Ank3 repression on CRMP2pT514 and whether it was modified by inhibition of GSK3 in our neuronal model system. While Ank3 exon 1b repression in neuro-2a cells resulted in no change in total CRMP2 levels compared to control cells $(P>0.05)$, inhibitory CRMP2-pT514 was significantly increased nearly 3-fold (Fig. 4e; post hoc vehicle-control vs vehiclerepressor $P<0.01)$. As expected, treatment with lithium $(1 \mathrm{mM})$ or CHIR99021 $(1 \mu \mathrm{M})$ attenuated the increase in CRMP2-pT514 induced by Ank3 exon $1 \mathrm{~b}$ repression, such that there was no significant difference between the Ank3repressed and control groups (Fig. 4e, post hoc lithiumcontrol vs lithium-repressor $P>0.05$, post hoc CHIR99021-control vs CHIR99021-repressor $P>0.05$ ). These results suggest that repression of brain-specific Ank3 increases EB3 expression and soluble:polymerized tubulin ratio through GSK3-mediated inhibition of CRMP2.

\section{Rescue of enhanced microtubule dynamics induced by Ank3 repression requires CRMP2 activity}

To address whether CRMP2 activity is required for GSK3 inhibition to rescue microtubule changes induced by $A n k 3$ repression, we evaluated whether lacosamide blocks the rescue by lithium or CHIR99021 in our neuronal model system. Lacosamide affects slow activation of sodium channels at doses of $100-500 \mu \mathrm{M}$, but lower doses of $3-5 \mu \mathrm{M}$ inhibit CRMP2 activity and tubulin binding without affecting sodium channel activation ${ }^{56-59}$. Pretreatment with a low $5 \mu \mathrm{M}$ dose of lacosamide $24 \mathrm{~h}$ prior to $1 \mathrm{mM}$ lithium or $1 \mu \mathrm{M}$ CHIR99021 treatment (Fig. 5a) blocked lithium and CHIR99021 from rescuing changes in EB3 (Fig. 5b) and steady state tubulin polymerization (Fig. 5c) induced by Ank3 exon $1 \mathrm{~b}$ repression. Specifically, when EB3 expression was evaluated by western blot, ANOVA and post hoc analysis revealed that, in the vehicle treated groups, Ank3 repression compared to control increased EB3 expression (Fig. 5b, vehicle-vehicle control vs vehicle-vehicle repressor $P<0.01$ ), which was rescued by treatment with lithium or CHIR99021 (Fig. 5b, vehiclelithium control vs vehicle-lithium repressor $P>0.05$, vehicle-CHIR99021 control vs vehicle-CHIR99021 repressor $P>0.05$ ). In contrast, in the lacosamide pretreated groups, Ank3 repression increased EB3 compared to control (Fig. 5b, lacosamide-vehicle control vs lacosamide-vehicle repressor $P<0.05$ ), but the increase was not rescued by lithium or CHIR99021 (Fig. 5b, lacosamide-lithium control vs lacosamide-lithium repressor $P<0.05$, lacosamide-CHIR99021 control vs lacosamide-CHIR99021 repressor $P<0.01)$. Similarly, when the ratio of soluble:polymerized tubulin was assessed, ANOVA and post hoc analysis determined that, in the vehicle treated groups, Ank3 repression increased the ratio of soluble:polymerized tubulin (Fig. 5c, vehiclevehicle control vs vehicle-vehicle repressor $P<0.05$ ), which was rescued by lithium or CHIR99021 (Fig. 5c, vehicle-lithium control vs vehicle-lithium repressor $P>$ 0.05 , vehicle-CHIR99021 control vs vehicle-CHIR99021 repressor $P>0.05$ ). In contrast, in the lacosamide pretreated groups, Ank3 repression increased the ratio of soluble:polymerized tubulin (Fig. 5c, lacosamide-vehicle control vs lacosamide-vehicle repressor $P<0.05$ ), but the increase was not rescued by lithium or CHIR99021 (Fig. $5 \mathrm{c}$, lacosamide-lithium control vs lacosamide-vehicle repressor $P<0.01$, lacosamide-CHIR99021 control vs lacosamide-CHIR99021 repressor $P<0.01$ ). Together, these data indicate that CRMP2 activity is required for GSK3 inhibition to rescue enhanced microtubule dynamics associated with repression of brain-specific Ank3.

\section{Discussion}

The current study used a multifaceted approach to identify and characterize the molecular functions of brainspecific Ank3. The key finding was that brain-specific Ank3 is associated with microtubule dynamics via a GSK3/CRMP2-dependent mechanism (Fig. 6). Our transcriptome-wide RNAseq analysis of Ank3+/- mouse hippocampus identified significantly altered expression of genes involved in pathways related to microtubule regulation and function, specifically axonal guidance signaling, microtubule dynamics, vesicle fusion, cytoskeletal organization, and extension of cellular protrusions. Subsequent live-cell imaging of primary neuron axons determined that microtubule dynamics are altered (i.e. decreased EB3 comet length and duration) in Ank3+/mice compared to $A n k 3+/+$ mice. To examine the underlying molecular and biochemical basis, we utilized a CRISPR/dCas9-based neuronal model of brain-specific Ank3 repression that exhibited changes in microtubule characteristics (increased EB3, increased ratio of soluble: polymerized tubulin, and decreased tubulin acetylation). The microtubule changes were rescued by inhibition of GSK3 and required active CRMP2, a GSK3 substrate that functions in microtubule stabilization. Taken together, this is the first study to demonstrate that brain-specific Ank3 has a vital role in microtubule dynamics via a GSK3/ CRMP2-dependent mechanism. Although it is not known whether ANK3 contributes to psychiatric illness by altering microtubule function, it is intriguing that microtubules and microtubule regulators have previously been implicated in psychiatric disorders ${ }^{20}$. Notably, microtubules are shortened and microtubule organization is perturbed in neuronal precursor cells from $\mathrm{BD}$ and 

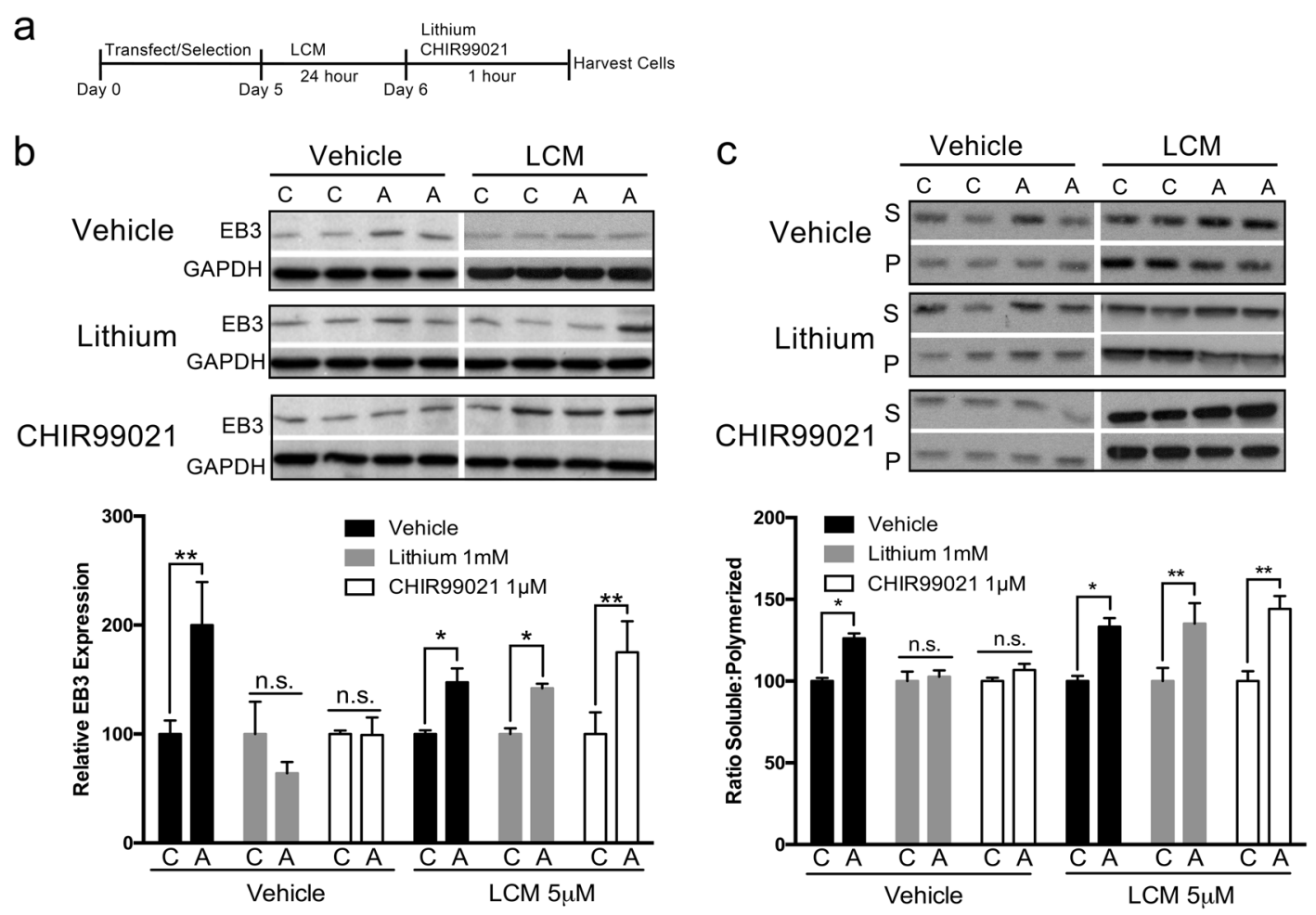

Fig. 5 Rescue of altered EB3 expression and tubulin polymerization induced by brain-specific Ank3 repression is blocked by inhibition of CRMP2. a Schematic representation of the experimental design. Mouse neuro-2a cells were transfected with pHAGE-EF1a-dCas9-KRAB repressor plasmid and sgRNA(MS2)_EF1a plasmid expressing either the non-targeting control sgRNA or the sgRNA targeting Ank3 exon 1b, followed by puromycin and zeocin selection. Cells were subsequently treated with $5 \mu \mathrm{M}$ lacosamide (LCM) for $24 \mathrm{~h}$, followed by treatment with lithium (1 mM) or CHIR99021 (1 $\mu \mathrm{M})$ for $1 \mathrm{~h}$, and cell harvest for protein extraction. b Top: Representative western blot of EB3 and GAPDH. Bottom: Quantification of EB3 expression normalized to GAPDH. Univariate ANOVA, LCM effect $F_{(1,60)}=2.00 P=0.163$, lithium/CHIR99021 effect $F_{(5,60)}=3.586 P=0.03$, Ank3 repression effect $F_{(1,60)}=21.389 P<0.001$, LCM and lithium/CHIR99021 interaction $F_{(2,60)}=7.505 P=0.001$. c Top: Representative western blot of atubulin in soluble $(S)$ and polymerized $(P)$ protein fractions. Bottom: Quantification of the ratio of soluble:polymerized tubulin. Univariate ANOVA, LCM effect $F_{(1,60)}=17.561 P<0.001$, lithium/CHIR99021 effect $F_{(5,60)}=0.203 P=0.817$, Ank3 repression effect $F_{(1,60)}=68.479 P<0.001$, LCM and lithium/ CHIR99021 interaction $F_{(2,60)}=6.113 P=0.004$. Western blot data were averaged from two independent experiments with three biological replicates per group in each experiment. The data were analyzed using two-tailed Student's $t$ test or ANOVA and Bonferroni post hoc tests. Data are presented as mean \pm s.e.m. ${ }^{*} P<0.05,{ }^{* *} P<0.01$. n.s indicates not significant, $C$ control sgRNA, A Ank3-targeting sgRNA

schizophrenic patients, respectively ${ }^{60}$. It will be important to investigate the relationship between $A N K 3$, microtubules, and psychiatric illness in future studies.

Live-cell imaging analyses determined that EB3 comet speed was unchanged in Ank3+l- mouse primary neuron axons, indicating that the rate of tubulin polymerization is normal. This suggests that tubulin heterodimers are able to form at microtubule plus ends when brain-specific Ank3 is reduced. However, the observed decrease in length and duration of EB3 comets in Ank3+/- mouse primary neurons suggests impaired stability of growing microtubules. This is characteristic of increased microtubule catastrophes ${ }^{61,62}$, i.e., switching from growth to rapid shortening, whereby the plus end stabilizing cap, where EB3 binds, is lost ${ }^{63}$. The shift in tubulin equilibrium to a soluble state induced by $A n k 3$ repression, as observed in our neuronal model system, provides additional evidence for increased catastrophes, since diminished microtubule elongation would lead to accumulation of tubulin in the soluble pool rather than incorporation into growing microtubules. In support of this, treatment with taxol to stabilize microtubules has been reported to reduce the pool of soluble tubulin ${ }^{41,64}$. Conversely, taxolresistant cancer cells have a lower proportion of polymerized tubulin, as well as increased microtubule dynamics as indicated by increased movement of EB3 comets $^{64}$. Furthermore, additional data from our neuronal model shows diminished tubulin acetylation after $A n k 3$ repression, suggesting that microtubules are more susceptible to rapid turnover. These studies support our findings that reduced expression of brain-specific Ank3 isoforms leads to increased activity at microtubule plus ends and instability of growing microtubules.

Among the lines of evidence implicating brain-specific Ank3 in regulation of microtubule dynamics, we found that reduction of brain-specific $A n k 3$ in mice and our 


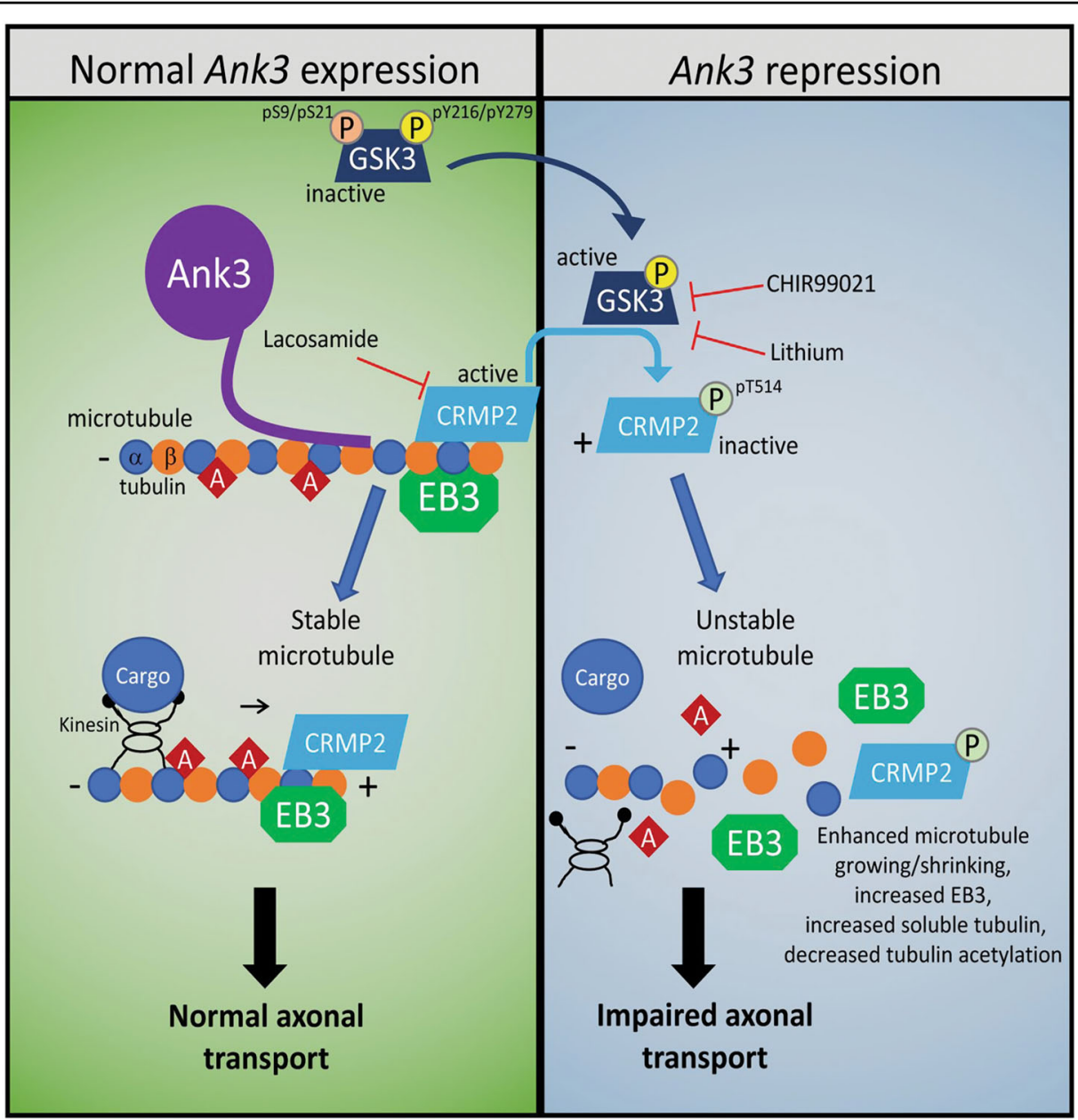

Fig. 6 Brain-specific Ank3 repression is associated with enhanced microtubule dynamics. Left: Under normal Ank3 expression, microtubules are stabilized by binding of microtubule-associated proteins (MAPs), such as EB3 and CRMP2, to the microtubule plus end where $\alpha$ - and $\beta$-tubulin heterodimers polymerize to facilitate elongation of the microtubule. As microtubules elongate, EB3 and CRMP2 move along the growing plus end tip to stabilize newly generated microtubule segments. Acetylation (red diamond) accumulates on a-tubulin within the microtubule due to low microtubule turnover. Motor proteins, such as kinesin, mediate transport of cellular cargo along the microtubule towards the plus end, which is oriented towards the distal axon. Right: Repression of brain-specific Ank3 reduces phosphorylation of GSK3 (pS9/pS21) and increases GSK3 activity, leading to an increase in CRMP2 phosphorylation (pT514) and impaired CRMP2 binding and stabilization of microtubules. Microtubules become more susceptible to catastrophes, as demonstrated by increased EB3 expression, reduced EB3 comet length and duration, and increased ratio of soluble:polymerized tubulin. The increased susceptibility to catastrophes increases microtubule turnover and decreases acetylation of a-tubulin. Inhibition of GSK3 activity by lithium or CHIR99021 reduces CRMP2 phosphorylation (pT514), thereby allowing CRMP2 to bind and stabilize microtubules. Pharmacological inhibition of CRMP2 by lacosamide reduces CRMP2 binding and stabilization of microtubules, which increases the amount of free tubulin and decreases acetylation of a-tubulin. Enhanced microtubule dynamics induced by brain-specific Ank3 repression may have a range of effects (e.g. axonal transport of synaptic vesicles, microtubule interaction with motor proteins) that alter neuronal function

neuronal model system increased the overall expression of the EB3 microtubule end-binding protein. This is consistent with a recent report that Ank3 knockdown in mouse primary hippocampal neurons increased EB3 puncta in axons ${ }^{65}$. However, that study reduced expression of all $A n k 3$ isoforms by $~ 90 \%$, whereas we targeted brain-specific Ank3 isoforms that are specifically implicated in psychiatric illness ${ }^{1-8}$, and we reduced expression by only $\sim 50 \%$, which is more consistent with patient brain expression changes ${ }^{12}$. While the precise mechanism by which EB3 expression is increased following brainspecific Ank3 repression is not known, several studies have shown that EB3 is elevated in response to enhanced microtubule dynamics ${ }^{66,67}$, providing support that brainspecific Ank3 repression is related to enhanced dynamics.

Our data suggest that $A n k 3$ reduction changes GSK3 and CRMP2 activity to modulate microtubule stability. Specifically, we found that repression of brain-specific Ank3 resulted in a reduction of GSK3 $\beta$-pS9 and GSK3 $\alpha$ pS21 (i.e. increased activity), and a concomitant increase 
in CRMP2-pT514 (i.e. decreased activity). This is interesting given our previous study demonstrating that lithium, which inhibits GSK3 in part through increasing pS9/pS21 levels through AKT activation ${ }^{50}$, reversed psychiatric-like behaviors in $A n k 3+/-$ mice $^{27}$. GSK3 has been previously implicated in psychiatric illness ${ }^{68-70}$ however, further studies are warranted to elucidate how GSK3 activity, Ank3, and microtubules interact to regulate psychiatric-like behaviors.

The GSK3 substrate CRMP2 regulates microtubule dynamics in at least two ways: by binding to tubulin heterodimers to enhance growth at microtubule plusends, and by serving as an adaptor between motor proteins and microtubules to promote microtubule elongation $^{33,54}$. In both cases, phosphorylation of CRMP2 T514 by GSK3 reduces the binding affinity of CRMP2 and destabilizes microtubules. In line with the observed elevation of GSK3 activity in Ank3-repressed neuro-2a cells, CRMP2-pT514 level was increased, supporting our hypothesis that changes in microtubule dynamics are mediated through a GSK3/CRMP2 pathway. We were able to substantiate this using a low dose of the CRMP2 antagonist lacosamide to inhibit tubulin polymerization $^{56-59}$, which prevented lithium and CHIR99021 from rescuing the microtubule changes in Ank3-repressed cells. Interestingly, CRMP2 activity has previously been implicated in the lithium responsiveness of BD patients ${ }^{22}$. In that study, cells derived from lithium-responsive patients had an elevated ratio of CRMP2-pT514 to total CRMP2 (i.e., decreased activity), similar to the elevated ratio we found in Ank3-repressed neuro-2a cells. This also falls in line with our observation that CRMP2 activity is required for lithium to rescue enhanced microtubule dynamics associated with $A n k 3$ repression.

Our findings that brain-specific Ank3 functions in microtubule dynamics advances our understanding of the role of ANK3 in supporting neuronal function, and potentially its contribution to psychiatric illness. Indeed, accumulating evidence suggests that abnormalities in the cytoskeleton are a potential mechanism for psychiatric illness via impaired microtubule-mediated axonal transport and synaptic plasticity ${ }^{60,71-74}$. It will be important to investigate how ANK3 influences microtubule-dependent processes in neurons and whether these processes underlie the association of $A N K 3$ with psychiatric illness.

\section{Acknowledgements}

The authors wish to thank Dr. Vann Bennett for providing the Ank3 mouse model, Dr. Feng Zhang for providing the sgRNA plasmid, and Francisca Meyer and Vivian Eijsink for advising on RNA sequencing data analysis. We thank Richard Bouley in the MGH Program in Membrane Biology (PMB) Microscopy Core for help with the confocal imaging. The Nikon A1R confocal in the PMB Microscopy Core was purchased using an $\mathrm{NIH}$ Shared Instrumentation Grant S10 RR031563-0. Additional support for the PMB Core came from the Boston Area Diabetes and Endocrinology Research Center (DK057521) and the MGH Center for the Study of Inflammatory Bowel Disease (DK043351). This research was supported by a Brain \& Behavior Research Foundation Independent Investigator award (TLP) and Young Investigator award (JCG), NIH grants R21 MH099760 (TLP), K22 NS094591 (JCG), and R01 MH107182 (PP), the Massachusetts General Hospital Executive Committee on Research (TLP and JCG), and the European Community's Seventh Framework Programme (FP7/ 2007-2013) under grant agreement $n^{\circ} 278948$ (TLP). This report reflects only the authors' views. The funding sponsors are not liable for any use that may be made of the information contained therein.

\section{Author details}

${ }^{1}$ Center for Genomic Medicine, Massachusetts General Hospital, Boston, MA, USA. ${ }^{2}$ Department of Psychiatry, Harvard Medical School, Boston, MA, USA.

${ }^{3}$ Stanley Center for Psychiatric Research, The Broad Institute of MIT and Harvard, Cambridge, MA, USA. ${ }^{4}$ Department of Biology, University of Massachusetts, Boston, MA, USA. ${ }^{5}$ Department of Psychology, Emmanuel College, Boston, MA, USA. ${ }^{6}$ The F.M. Kirby Neurobiology Center, Boston Children's Hospital, Harvard Medical School, Boston, MA, USA. 'Division of Medical Sciences, Harvard Medical School, Boston, MA, USA. ${ }^{8}$ Department of Physiology, Department of Psychiatry and Behavioral Sciences, Northwestern University Feinberg School of Medicine, Chicago, IL, USA. ${ }^{9}$ Department of Molecular Animal Physiology, Donders Institute for Brain, Cognition and Behaviour, Centre for Neuroscience, Faculty of Science, Radboud University, Nijmegen, The Netherlands. ${ }^{10}$ Department of Human Genetics, Radboud University Medical Center, Nijmegen, The Netherlands

\section{Conflict of interest}

The authors declare that they have no conflict of interest.

\section{Publisher's note}

Springer Nature remains neutral with regard to jurisdictional claims in published maps and institutional affiliations.

Supplementary Information accompanies this paper at (https://doi.org/ 10.1038/s41398-018-0182-y).

Received: 24 April 2018 Accepted: 11 May 2018

Published online: 25 July 2018

\section{References}

1. Ferreira, M. A. et al. Collaborative genome-wide association analysis supports a role for ANK3 and CACNA1C in bipolar disorder. Nat. Genet 40, 1056-1058 (2008).

2. Schulze, T. G. et al. Two variants in Ankyrin 3 (ANK3) are independent genetic risk factors for bipolar disorder. Mol. Psychiatry 14, 487-491 (2009).

3. Group PGCBDW. Large-scale genome-wide association analysis of bipolar disorder identifies a new susceptibility locus near ODZ4. Nat. Genet 43, 977-983 (2011)

4. Tesli, M. et al. Association analysis of ANK3 gene variants in nordic bipolar disorder and schizophrenia case-control samples. Am. J. Med. Genet. B Neuropsychiatr. Genet. 156B, 969-974 (2011).

5. Baum, A. E. et al. A genome-wide association study implicates diacylglycerol kinase eta (DGKH) and several other genes in the etiology of bipolar disorder. Mol. Psychiatry 13, 197-207 (2008).

6. $\mathrm{Bi}, \mathrm{C}$. et al. Mutations of ANK3 identified by exome sequencing are associated with autism susceptibility. Human. Mutat. 33, 1635-1638 (2012).

7. Iqbal, Z. et al. Homozygous and heterozygous disruptions of ANK3: at the crossroads of neurodevelopmental and psychiatric disorders. Human. Mol. Genet. 22, 1960-1970 (2013).

8. Kosmicki, J. A. et al. Refining the role of de novo protein-truncating variants in neurodevelopmental disorders by using population reference samples. Nat. Genet. 49, 504-510 (2017).

9. Shi, L. et al. Whole-genome sequencing in an autism multiplex family. Mol. Autism 4, 8 (2013).

10. Sanders, S. J. et al. De novo mutations revealed by whole-exome sequencing are strongly associated with autism. Nature 485, 237-241 (2012).

11. Lippard, E. T. et al. Effects of ANK3 variation on gray and white matter in bipolar disorder. Mol. Psychiatry 22, 1345-1351 (2016). 
12. Roussos, P. et al. Molecular and genetic evidence for abnormalities in the nodes of Ranvier in schizophrenia. Arch. Gen. Psychiatry 69, 7-15 (2012).

13. Rueckert, E. H. et al. Cis-acting regulation of brain-specific ANK3 gene expression by a genetic variant associated with bipolar disorder. Mol. Psychiatry 18, 922-929 (2013).

14. Nie, F. et al. Genetic analysis of SNPs in CACNA1C and ANK3 gene with schizophrenia: A comprehensive meta-analysis. Am. J. Med. Genet. B Neuropsychiatr. Genet. 168, 637-648 (2015).

15. Bennett, V. \& Baines, A. J. Spectrin and ankyrin-based pathways: metazoan inventions for integrating cells into tissues. Physiol. Rev. 81, 1353-1392 (2001).

16. Jenkins, S. M. \& Bennett, V. Ankyrin-G coordinates assembly of the spectrinbased membrane skeleton, voltage-gated sodium channels, and L1 CAMs at Purkinje neuron initial segments. J. Cell Biol. 155, 739-746 (2001).

17. Leussis, M. P., Madison, J. M. \& Petryshen, T. L. Ankyrin 3: genetic association with bipolar disorder and relevance to disease pathophysiology. Biol. Mood Anxiety Disord. 2, 18 (2012).

18. Zhou, D. et al. AnkyrinG is required for clustering of voltage-gated Na channels at axon initial segments and for normal action potential firing. J. Cell Biol. 143, 1295-1304 (1998).

19. Kordeli, E., Lambert, S. \& Bennett, V. AnkyrinG. A new ankyrin gene with neuralspecific isoforms localized at the axonal initial segment and node of Ranvier. J. Biol. Chem. 270, 2352-2359 (1995).

20. Marchisella, F., Coffey, E. T. \& Hollos, P. Microtubule and microtubule associated protein anomalies in psychiatric disease. Cytoskeleton (Hoboken) 73, 596-611 (2016).

21. Drago, A., Crisafulli, C., Sidoti, A., Calabro, M. \& Serretti, A. The microtubuleassociated molecular pathways may be genetically disrupted in patients with Bipolar Disorder. Insights from the molecular cascades. J. Affect Disord. 190, 429-438 (2016).

22. Tobe, B. T. D. et al. Probing the lithium-response pathway in hiPSCs implicates the phosphoregulatory set-point for a cytoskeletal modulator in bipolar pathogenesis. Proc. Natl Acad. Sci. USA 114, E4462-E4471 (2017).

23. Akhmanova, A. \& Steinmetz, M. O. Control of microtubule organization and dynamics: two ends in the limelight. Nat. Rev. Mol. Cell Biol. 16, 711-726 (2015).

24. Bennett, V. \& Davis, J. Erythrocyte ankyrin: immunoreactive analogues are associated with mitotic structures in cultured cells and with microtubules in brain. Proc. Natl Acad. Sci. USA 78, 7550-7554 (1981).

25. Davis, J. Q. \& Bennett, V. Brain ankyrin. A membrane-associated protein with binding sites for spectrin, tubulin, and the cytoplasmic domain of the erythrocyte anion channel. J. Biol. Chem. 259, 13550-13559 (1984).

26. Freal, A. et al. Cooperative Interactions between $480 \mathrm{kDa}$ Ankyrin- $\mathrm{G}$ and EB Proteins Assemble the Axon Initial Segment. J. Neurosci. 36, 4421-4433 (2016).

27. Gottschalk, M. G. et al. Lithium reverses behavioral and axonal transport-related changes associated with ANK3 bipolar disorder gene disruption. Eur. Neuropsychopharmacol. 27, 274-288 (2017).

28. Leussis, M. P. et al. The ANK3 bipolar disorder gene regulates psychiatricrelated behaviors that are modulated by lithium and stress. Biol. Psychiatry $\mathbf{7 3}$ 683-690 (2013).

29. Prickaerts, J. et al. Transgenic mice overexpressing glycogen synthase kinase 3beta: a putative model of hyperactivity and mania. J. Neurosci. 26, 9022-9029 (2006).

30. Polter, A. et al. Deficiency in the inhibitory serine-phosphorylation of glycogen synthase kinase-3 increases sensitivity to mood disturbances. Neuropsychopharmacology 35, 1761-1774 (2010).

31. Chae, Y. C. et al. Collapsin response mediator protein-2 regulates neurite formation by modulating tubulin GTPase activity. Cell Signal 21, 1818-1826 (2009).

32. Charrier, E. et al. Collapsin response mediator proteins (CRMPs): involvement in nervous system development and adult neurodegenerative disorders. Mol. Neurobiol. 28, 51-64 (2003).

33. Fukata, $Y$. et al. CRMP-2 binds to tubulin heterodimers to promote microtubule assembly. Nat. Cell Biol. 4, 583-591 (2002).

34. Yoshimura, T. et al. GSK-3beta regulates phosphorylation of CRMP-2 and neuronal polarity. Cell 120, 137-149 (2005).

35. Maniar, T. A. et al. UNC-33 (CRMP) and ankyrin organize microtubules and localize kinesin to polarize axon-dendrite sorting. Nat. Neurosci. 15, 48-56 (2012).

36. Trapnell, C. et al. Differential gene and transcript expression analysis of RNAseq experiments with TopHat and Cufflinks. Nat. Protoc. 7, 562-578 (2012).
37. Pekkurnaz, G., Trinidad, J. C., Wang, X., Kong, D. \& Schwarz, T. L. Glucose regulates mitochondrial motility via Milton modification by O-GICNAC transferase. Cell 158, 54-68 (2014).

38. Konermann, S. et al. Genome-scale transcriptional activation by an engineered CRISPR-Cas9 complex. Nature 517, 583-588 (2015).

39. Kearns, N. A. et al. Cas9 effector-mediated regulation of transcription and differentiation in human pluripotent stem cells. Development 141, 219-223 (2014).

40. Untergasser, A. et al. Primer3Plus, an enhanced web interface to Primer3. Nucleic Acids Res. 35, W71-W74 (2007).

41. Sharma, N., Kosan, Z. A., Stallworth, J. E., Berbari, N. F. \& Yoder, B. K. Soluble levels of cytosolic tubulin regulate ciliary length control. Mol. Biol. Cell 22 806-816 (2011).

42. Tokesi, N. et al. TPPP/p25 promotes tubulin acetylation by inhibiting histone deacetylase 6. J. Biol. Chem. 285, 17896-17906 (2010).

43. Akhmanova, A. \& Steinmetz, M. O. Microtubule + TIPs at a glance. J. Cell Sci. 123, 3415-3419 (2010).

44. Stepanova, T. et al. Visualization of microtubule growth in cultured neurons via the use of EB3-GFP (end-binding protein 3-green fluorescent protein). J. Neurosci. 23, 2655-2664 (2003).

45. Hubbert, C. et al. HDAC6 is a microtubule-associated deacetylase. Nature $\mathbf{4 1 7}$ 455-458 (2002)

46. Palazzo, A., Ackerman, B. \& Gundersen, G. G. Cell biology: Tubulin acetylation and cell motility. Nature 421, 230 (2003).

47. Fourest-Lieuvin, A. et al. Microtubule regulation in mitosis: tubulin phosphorylation by the cyclin-dependent kinase Cdk1. Mol. Biol. Cell 17, 1041-1050 (2006).

48. Giannakakou, P. et al. A common pharmacophore for epothilone and taxanes: molecular basis for drug resistance conferred by tubulin mutations in human cancer cells. Proc. Natl Acad. Sci. USA 97, 2904-2909 (2000).

49. Kamal, A. et al. Synthesis of terphenyl benzimidazoles as tubulin polymerization inhibitors. Eur. J. Med Chem. 50, 9-17 (2012).

50. Pan, J. Q. et al. AKT kinase activity is required for lithium to modulate moodrelated behaviors in mice. Neuropsychopharmacology 36, 1397-1411 (2011).

51. Stambolic, V. \& Woodgett, J. R. Mitogen inactivation of glycogen synthase kinase-3 beta in intact cells via serine 9 phosphorylation. Biochem J. 303 (Pt 3), 701-704 (1994).

52. Sutherland, C., Leighton, I. A. \& Cohen, P. Inactivation of glycogen synthase kinase-3 beta by phosphorylation: new kinase connections in insulin and growth-factor signalling. Biochem J. 296 (Pt 1), 15-19 (1993).

53. Hughes, K., Nikolakaki, E., Plyte, S. E., Totty, N. F. \& Woodgett, J. R. Modulation of the glycogen synthase kinase-3 family by tyrosine phosphorylation. EMBO J. 12, 803-808 (1993).

54. Kawano, Y. et al. CRMP-2 is involved in kinesin-1-dependent transport of the Sra-1MAVE1 complex and axon formation. Mol. Cell Biol. 25, 9920-9935 (2005).

55. Cole, A. R. et al. Distinct priming kinases contribute to differential regulation of collapsin response mediator proteins by glycogen synthase kinase-3 in vivo. J. Biol. Chem. 281, 16591-16598 (2006).

56. Errington, A. C., Coyne, L., Stohr, T., Selve, N. \& Lees, G. Seeking a mechanism of action for the novel anticonvulsant lacosamide. Neuropharmacology 50, 1016-1029 (2006).

57. Errington, A. C., Stohr, T., Heers, C. \& Lees, G. The investigational anticonvulsant lacosamide selectively enhances slow inactivation of voltage-gated sodium channels. Mol. Pharmacol. 73, 157-169 (2008).

58. Wilson, S. M. \& Khanna, R. Specific binding of lacosamide to collapsin response mediator protein 2 (CRMP2) and direct impairment of its canonical function: implications for the therapeutic potential of lacosamide. Mol. Neurobiol. 51, 599-609 (2015).

59. Wilson, S. M. et al. The functionalized amino acid (S)-Lacosamide subverts CRMP2-mediated tubulin polymerization to prevent constitutive and activitydependent increase in neurite outgrowth. Front Cell Neurosci. 8, 196 (2014).

60. Solis-Chagoyan, $H$. et al. Microtubule organization and L-type voltage-activated calcium current in olfactory neuronal cells obtained from patients with schizophrenia and bipolar disorder. Schizophr. Res 143, 384-389 (2013).

61. Leterrier, C. et al. Ankyrin G membrane partners drive the establishment and maintenance of the axon initial segment. Front Cell Neurosci. 11, 6 (2017).

62. Stone, M. C., Nguyen, M. M., Tao, J., Allender, D. L. \& Rolls, M. M. Global upregulation of microtubule dynamics and polarity reversal during regeneration of an axon from a dendrite. Mol. Biol. Cell 21, 767-777 (2010). 
63. Maurer, S. P., Fourniol, F. J., Bohner, G., Moores, C. A. \& Surrey, T. EBs recognize a nucleotide-dependent structural cap at growing microtubule ends. Cell 149, 371-382 (2012).

64. McGrail, D. J. et al. Alterations in ovarian cancer cell adhesion drive taxol resistance by increasing microtubule dynamics in a FAK-dependent manner. Sci. Rep. 5, 9529 (2015)

65. Leterrier, $C$. et al. End-binding proteins EB3 and EB1 link microtubules to ankyrin $\mathrm{G}$ in the axon initial segment. Proc. Natl Acad. Sci. USA 108, 8826-8831 (2011).

66. Komarova, Y. et al. Mammalian end binding proteins control persistent microtubule growth. J. Cell Biol. 184, 691-706 (2009).

67. Mohan, R. et al. End-binding proteins sensitize microtubules to the action of microtubule-targeting agents. Proc. Natl Acad. Sci. USA 110, 8900-8905 (2013).

68. de Sousa, R. T. et al. Lithium increases platelet serine-9 phosphorylated GSK3beta levels in drug-free bipolar disorder during depressive episodes. J. Psychiatr. Res. 62, 78-83 (2015).
69. Jimenez, E. et al. Association between GSK3beta gene and increased impulsivity in bipolar disorder. Eur. Neuropsychopharmacol. 24, 510-518 (2014).

70. Jope, R. S. \& Roh, M. S. Glycogen synthase kinase-3 (GSK3) in psychiatric diseases and therapeutic interventions. Curr. Drug Targets 7, 1421-1434 (2006).

71. English, J. A., Dicker, P., Focking, M., Dunn, M. J. \& Cotter, D. R. 2-D DIGE analysis implicates cytoskeletal abnormalities in psychiatric disease. Proteomics $\mathbf{9}$ 3368-3382 (2009)

72. Goldstein, L. S. Axonal transport and neurodegenerative disease: can we see the elephant? Prog. Neurobiol. 99, 186-190 (2012).

73. Shao, L., Golbaz, K., Honer, W. G. \& Beasley, C. L. Deficits in axon-associated proteins in prefrontal white matter in bipolar disorder but not schizophrenia. Bipolar Disord. 18, 342-351 (2016).

74. Woolf, N. J., Craddock, T. J. A., Friesen, D. E. \& Tuszynski, J. A. Neuropsycchiatric Illness: a case for impaired neuroplasticity and possible quantum processing derailment in microtubules. NeruoQuantology 8, 13-28 (2010). 